\title{
Clinical value of serum tumor markers CA19-9, CA125 and CA72-4 in the diagnosis of pancreatic carcinoma
}

\author{
ZI WANG ${ }^{1,2}$ and YA-PING TIAN ${ }^{1}$ \\ ${ }^{1}$ Department of Clinical Biochemistry, Chinese PLA General Hospital, Beijing 100853; \\ ${ }^{2}$ Medical School of Nankai University, Tianjin 300071, P.R. China
}

Received October 26, 2013; Accepted December 9, 2013

DOI: $10.3892 / \mathrm{mco} .2013 .226$

\begin{abstract}
CA125 and CA72-4 are members of a family of high-molecular weight glycosylated proteins and are commonly considered as biomarkers in the diagnosis of ovarian and gastric cancer, respectively. However, recent studies have revealed that these two markers may be of clinical value in the diagnosis of pancreatic cancer. As the availability of data regarding CA72-4 and CA125 in the diagnosis of pancreatic cancer is limited, the aim of this study was to investigate the clinical value of serum tumor markers CA19-9, CA125 and CA72-4 in the diagnosis of pancreatic carcinoma according to logistic regression analysis and receiver operating characteristic (ROC) curves and to investigate the correlation of these markers with tumor TNM stage and location. An immunoradiometric assay was used to measure pre-operative serum CA19-9, CA125 and CA72-4 levels in 75 patients with pancreatic carcinoma and 70 patients with benign pancreatic diseases. The concentrations of serum CA19-9, CA125 and CA72-4 in patients with pancreatic carcinoma were found to be significantly higher $(\mathrm{P}<0.05)$ compared with those with benign pancreatic diseases. The combined detection of two serum markers (CA19-9 + CA72-4) yielded a ROC value of 0.895 that was significantly higher compared to others $(\mathrm{P}<0.05)$ in distinguishing pancreatic cancer from benign pancreatic diseases. At optimal cut-off, the sensitivity and specificity of combined detection (CA19-9 + CA72-4) were 70.6 and 92.8\%, respectively. The concentrations of CA125 and CA19-9 in patients with pancreatic adenocarcinoma were found to be significantly higher $(\mathrm{P}<0.05)$ compared with those of pancreatic neuroendocrine carcinoma. In conclusion, the combined detection of CA19-9 and CA72-4) may significantly improve
\end{abstract}

Correspondence to: Professor Ya-Ping Tian, Department of Clinical Biochemistry, Chinese PLA General Hospital, 28 Fuxing Road, Beijing 100853, P.R. China

E-mail: tianyp61@gmail.com; tianyp@301hospital.com.cn

Abbreviations: ROC, receiver operating characteristic; AUC, area under the ROC curve

Key words: pancreatic cancer, combined detection, receiver operating characteristic curve the diagnostic specificity and the serum concentrations of CA125 and CA19-9 are correlated with tumor histological type.

\section{Introduction}

Pancreatic cancer is the fourth leading cause of cancer-related mortality in USA (1) and is a highly lethal disease, characterized by difficulty of diagnosis, distant metastasis and aggressive local invasion at an early stage (2). The 5-year survival rate of pancreatic cancer was reported to be $<5.5 \%$ (3-5) and almost all the patients succumbed to their disease within 2 years (6). Therefore, in order to improve prognosis, the key determinant is accurate diagnosis at an early stage. The detection of serum tumor markers was shown to be an effective and non-invasive diagnostic tool, with $>5$ serum tumor markers for the diagnosis of pancreatic cancer. It was recently suggested that CA125 and CA72-4 $(7,8)$ were potentially of clinical value for the diagnosis of pancreatic cancer, although they were commonly considered as biomarkers for the diagnosis of ovarian and gastric cancer, respectively. However, in practice, there was inadequate availability of information regarding the use of CA125 and CA72-4 for the diagnosis of pancreatic cancer and it was hypothesized that the combined detection of these markers may increase their diagnostic sensibility or specificity. Receiver operating characteristic (ROC) curves $(9,10)$ have been widely used as a standard approach for calculating the sensitivity and specificity of medical diagnostic tests. In this study, we aimed to investigate the diagnostic sensitivity and specificity of single and combined marker detection by ROC and evaluate the correlations between serum markers and clinically relevant parameters.

\section{Materials and methods}

Study population. Between January, 2012 and June, 2013, data were collected on 75 patients ( 31 females and 44 males) with pancreatic cancer, with a median age of 57 years (range, 25-80 years) and 70 patients with benign pancreatic diseases (27 women and 43 men), with a median age of 47 years (range, 26-73 years) in the PLA General Hospital, Beijing, China. All diagnoses were confirmed by at least one of the following: intraoperative biopsy examination, pathological examination, or radiological detection. According to the fifth edition of the 
Table I. Serum concentrations of CA19-9, CA125 and CA72-4 in different patient groups [median $\left.\left(\mathrm{Q}_{1-3}\right)\right]$.

\begin{tabular}{llcr}
\hline $\begin{array}{l}\text { Groups } \\
\text { ml) }\end{array}$ & CA125 $(\mathrm{ng} / \mathrm{ml})$ & CA19-9 $(\mathrm{ng} / \mathrm{ml})$ & CA72-4 $(\mathrm{ng} /$ \\
\hline Pancreatic carcinoma & $34.1(12.3,164.8)^{\mathrm{a}}$ & $414.6(60.6,3132.0)^{\mathrm{a}}$ & $2.8(1.7,15.3)^{\mathrm{a}}$ \\
Benign pancreatic diseases & $17.9(10.7,87.4)$ & $14.3(7.8,57.0)$ & $1.2(1.0,1.9)$ \\
\hline
\end{tabular}

${ }^{a} \mathrm{P}<0.05$ compared with benign pancreatic diseases.

Table II. Performance characteristics of serum biomarkers for distinguishing pancreatic cancer from benign pancreatic diseases.

\begin{tabular}{lcccc}
\hline Markers & ROC area $(\%)$ & $95 \%$ CI & Sensitivity $(\%)$ & Specificity $(\%)$ \\
\hline CA19-9 & $83.8^{\mathrm{a}}$ & $(76.8-89.4)$ & 73.3 & 85.7 \\
CA125 & $60.2^{\mathrm{a}}$ & $(51.7-68.2)$ & 30.7 & 88.6 \\
CA72-4 & $78.7^{\mathrm{a}}$ & $(71.2-85.1)$ & 71.4 & 87.1 \\
CA19-9 + CA72-4 & 89.5 & $(83.3-94.0)$ & 70.6 & 92.8
\end{tabular}

${ }^{\mathrm{a}} \mathrm{P}<0.05$ compared with CA19-9 + CA72-4. ROC, receiver operating characteristic; CI, confidence interval.

TNM classification (11), there were 13 stage I, 8 stage II and 54 stage III-IV patients. The location of the tumor was divided into head and body/tail. The tumor size was divided into $\leq 5$ or $>5 \mathrm{~cm}$ in diameter. Serum samples were collected prior to operation or chemotherapy and the patients provided informed consent for the analysis of CA125, CA19-9 and CA72-4. This study was approved by the Medical Ethics Committee of the PLA General Hospital (Beijing, China).

Evaluation of tumor markers. The serum CA19-9, CA125 and CA72-4 levels were measured using the electrochemiluminescence immunoassay on the Roche cobas 6000 analyzer (Roche Diagnostics, Mennheim, Germany). The recommended cut-off values for diagnostic purposes were $37 \mathrm{U} / 1$ for CA19-9, $33 \mathrm{U} / 1$ for CA125 and $10 \mathrm{U} / 1$ for CA72-4.

Statistical analysis. The Mann-Whitney U test (SPSS 17.0 statistical software package for Windows; SPSS Inc., Chicago, IL, USA) was applied to determine the differences in marker levels, followed by a logistic regression analysis to identify the related serum markers in the diagnosis of pancreatic cancer and yield a logistic equation and an ROC curve. The MedCalc statistical software package (MedCalc Software, Mariakerke, Belgium) was to assess the difference between different areas under the curve (AUC) and to evaluate the diagnostic sensitivity and specificity at the optimal cut-off. The data was described by $\mathrm{Q}_{1-3}$. $\mathrm{P}<0.05$ was considered to indicate a statistically significant difference.

\section{Results}

Serum concentrations of CA19-9, CA125 and CA72-4 in different groups. The concentrations of CA19-9, CA125 and CA72-4 in patients with pancreatic carcinoma were significantly higher $(\mathrm{P}<0.05)$ compared with those in patients with benign pancreatic diseases (Table I).

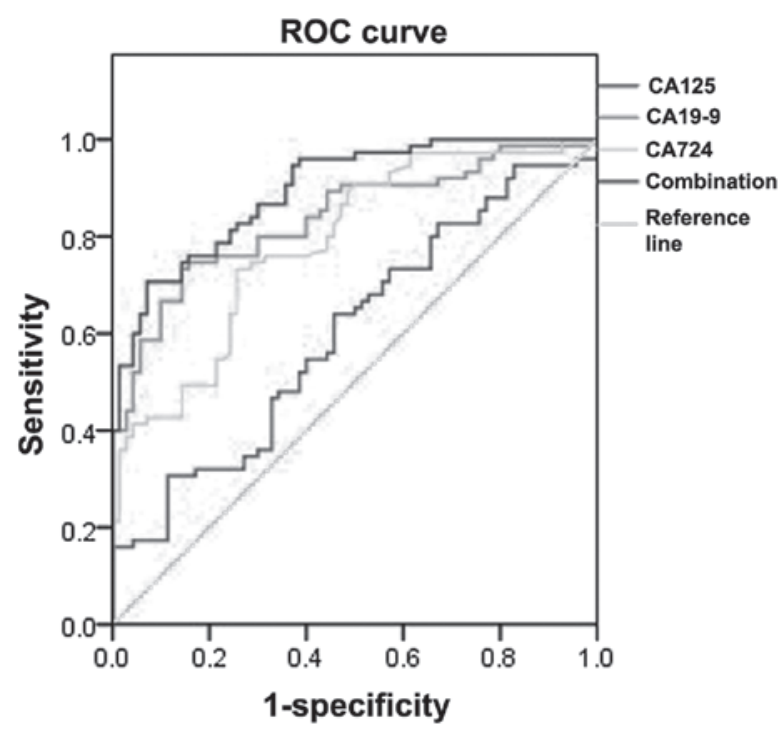

Figure 1. Receiver operating characteristic (ROC) curve of single and combined marker detection in distinguishing pancreatic cancer from benign pancreatic diseases.

Value of serum CA19-9, CA125 and CA72-4 single and combined detection in the diagnosis of pancreatic cancer. Logistic regression analysis was performed to identify related serum markers; subsequently, CA19-9 $(\mathrm{P}<0.05)$ and CA72-4 $(\mathrm{P}<0.05)$ were selected into the logistic regression equation $\left(\mathrm{y}=1.496-0.004 \mathrm{x}_{\mathrm{CA} 19-9}-0.207 \mathrm{x}_{\mathrm{CA72-4}}, \mathrm{P}<0.001\right)$. In terms of AUC, the combined detection of CA19-9 and CA72-4 was significantly higher $(\mathrm{P}<0.05)$ compared to that of other markers. The maximum corresponding sensitivity and specificity was yielded at the optimal cut-off point and the sensitivity of CA19-9 was the highest among marker detection, whereas the specificity of the combined detection of CA19-9 and CA72-4) was higher compared to others (Table II and Fig. 1). 
Table III. Association between serum markers and clinically related parameters of pancreatic cancer $\left[\right.$ median $\left.\left(\mathrm{Q}_{1-3}\right)\right]$.

\begin{tabular}{|c|c|c|c|c|c|c|c|}
\hline \multirow[b]{2}{*}{ Parameters } & \multirow[b]{2}{*}{ No. } & \multicolumn{2}{|l|}{ CA125 } & \multicolumn{2}{|l|}{ CA19-9 } & \multicolumn{2}{|c|}{ CA72-4 } \\
\hline & & Value (ng/ml) & P-value & Value (ng/ml) & P-value & Value (ng/ml) & P-value \\
\hline \multicolumn{8}{|l|}{ Histological type } \\
\hline Adenocarcinoma & 62 & $46.4(14.4-186.3)$ & & $649.3(132.9-3783.0)$ & & $3.4(1.7-16.4)$ & \\
\hline $\begin{array}{l}\text { Neuroendocrine } \\
\text { carcinoma }\end{array}$ & 13 & $15.0(9.7-58.4)$ & $<0.05$ & $18.2(7.7-107.7)$ & $<0.05$ & $2.2(1.8-4.5)$ & 0.291 \\
\hline \multicolumn{8}{|l|}{ Location } \\
\hline Head & 42 & $33.6(12.3-169.3)$ & & $412.4(100.8-2237.5)$ & & $2.7(1.5-15.8)$ & \\
\hline Body/tail & 33 & $38.0(12.3-162.0)$ & 0.835 & $415.6(36.4-3665.5)$ & 0.658 & $3.4(1.8-12.7)$ & 0.806 \\
\hline \multicolumn{8}{|l|}{ TNM stage } \\
\hline I & 13 & $11.7(10.0-85.9)$ & & $53.3(13.4-1107.3)$ & & $2.1(1.1-3.6)$ & \\
\hline II & 8 & $38.5(11.3-183.1)$ & & $226.1(78.7-3623.7)$ & & $3.4(2.1-15.3)$ & \\
\hline III-IV & 54 & $53.5(17.2-532.3)$ & 0.064 & $492.6(81.4-4653.7)$ & 0.386 & $8.1(1.8-122.8)$ & 0.082 \\
\hline \multicolumn{8}{|l|}{ Size $(\mathrm{cm})$} \\
\hline$\leq 5$ & 18 & $14.5(10.0-57.0)$ & & $151.1(27.2-935.6)$ & & $2.4(1.6-6.42)$ & \\
\hline$>5$ & 26 & $20.9(13.2-141.6)$ & 0.346 & $348.8(47.0-1594.5)$ & 0.28 & $2.8(1.4-18.0)$ & 0.702 \\
\hline
\end{tabular}

Parenthetical data, $\mathrm{Q}_{1}$ (the first quartile) and $\mathrm{Q}_{3}$ (the third quartile).

Association between serum markers and clinically related parameters of pancreatic cancer. The concentrations of CA125 and CA19-9 in patients with pancreatic adenocarcinoma were found to be significantly higher $(\mathrm{P}<0.05)$ compared with those with pancreatic neuroendocrine carcinoma. By contrast, as regards the levels of CA72-4, there was no significant difference $(\mathrm{P}>0.05)$ between pancreatic adenocarcinoma and pancreatic neuroendocrine carcinoma. In terms of tumor location, the concentrations of CA125, CA19-9 and CA72-4 were not significantly different $(\mathrm{P}>0.05)$ between head and body/tail. The levels of CA125, CA19-9 and CA72-4 in stage III-IV patients were found to be higher compared with those in stage II patients, although the difference was not significant (Table III).

\section{Discussion}

As previously described, serological markers that were in essence antigens and bioactive materials from tumor tissues, minimally produced by normal cells (12), were commonly used for the diagnosis of pancreatic carcinoma, particularly CA19-9, an intracellular adhesion molecule with a molecular weight of $>500000$, that was widely applied in the identification of pancreatic cancer. In a previous study by Goonetilleke and Siriwardena (13), a systematic review of the MEDLINE database revealed that the median sensitivity of CA19-9 for diagnosing pancreatic cancer was 79 , whereas the median specificity was $82 \%$. Additionally, in a previous trial by Ni et al (14), the sensitivity of CA19-9 for the diagnosis of pancreatic cancer was $\sim 80$, whereas the specificity was merely $43 \%$. Moreover, in a study by Ma et al (12), it was demonstrated that the sensitivity and specificity of CA19-9 for the diagnosis of pancreatic cancer were 79.49 and $95.31 \%$, respectively.
Our study demonstrated that the concentration of CA19-9 in patients with pancreatic adenocarcinoma was significantly higher compared to that in pancreatic neuroendocrine carcinoma patients. Although the concentrations of CA19-9, CA125 and CA72-4 in stage III-IV were higher compared to those in stage II patients, the differences were not significant.

In 2001, CA125 (mucin 16) was identified by molecular cloning (15) as a cell surface glycoprotein that could be involved in promoting ovarian cancer cell growth. It was originally considered as a specific biological marker for ovarian cancer; however, with an increasing number of studies, it was demonstrated that it could also play an important role in diagnosing different types of cancer, including gastric, colorectal and pancreatic carcinoma. Furthermore, CA125 was found to be an independent predictor of poor outcome in pancreatic ductal adenocarcinoma and was strongly associated with patient survival rate (16). It was suggested that the concentrations of CA19-9 and CA125 were correlated with tumor size, clinical stage and tumor differentiation (17). However, in our study, the levels of serum CA125, CA19-9 and CA72-4 were not strongly affected by tumor size and although the specificity of the combined detection (CA19-9 + CA72-4) was increased to $92.8 \%$, its sensitivity was inferior to that of CA19-9.

In conclusion, our findings suggested the specificity of the combined detection of CA19-9 and CA72-4) was increased to $92.8 \%$, suggesting it may be a valuable strategy for pancreatic cancer screening at an early stage. In terms of sensitivity, the CA19-9 was found to be a reliable biomarker for the diagnosis of pancreatic cancer. Furthermore, the concentrations of CA125 and CA19-9 were found to be significantly different between pancreatic adenocarcinoma and neuroendocrine carcinoma. 


\section{Acknowledgements}

This study was supported by grant no. 2011AA02A111 from the National High Technology Research and Development Program of China (863 Program).

\section{References}

1. Jemal A, Siegel R, Ward E, et al: Cancer statistics, 2008 CA Cancer J Clin 58: 71-96, 2008.

2. Postier RG: The challenge of pancreatic cancer. Am J Surg 186: $579-582,2003$

3. Kato K, Kamada H, Fujimori T, Aritomo Y, Ono M and Masaki T: Molecular biologic approach to the diagnosis of pancreatic carcinoma using specimens obtained by EUS-guided fine needle aspiration. Gastroenterol Res Pract 2012: 243524, 2012.

4. Li D, Xie K, Wolff R and Abbruzzese JL: Pancreatic cancer Lancet 363: 1049-1057, 2004.

5. Gudjonsson B: Carcinoma of the pancreas: critical analysis of costs, results of resections, and the need for standardized reporting. J Am Coll Surg 181: 483-503, 1995.

6. Niederhuber JE, Brennan MF and Menck HR: The national cancer data base report on pancreatic cancer. Cancer 76 1671-1677, 1995

7. Haridas D, Chakraborty S, Ponnusamy MP, et al: Pathobiological implications of MUC16 expression in pancreatic cancer. PLoS One 6: e26839, 2011.

8. Ding YL, Zhao YB, Rong Q and Lu XM: Analysis on high level of serum CA72-4 in patients with malignant tumors in 187 cases. Lab Med Clin 7: 1528, 2010 (In Chinese).
9. Zou KH, O'Malley AJ and Mauri L: Receiver-operating characteristic analysis for evaluating diagnostic tests and predictive models. Circulation 115: 654-657, 2007.

10. Walter SD and Sinuff T: Studies reporting ROC curves of diagnostic and prediction data can be incorporated into meta-analyses using corresponding odds ratios. J Clin Epidemiol 60: 530-534, 2007.

11. Sobin L and Wittekind C (eds): TNM: Classification of Malignant Tumours. 5th edition. Wiley-Liss, New York, NY, 1997.

12. Ma Z, Ma Q and Wang Z: An evaluation of the diagnostic value of CA19-9 and CEA levels in patients with pancreatic cancer. J Nanjing Med Univ 23: 199-202, 2009.

13. Goonetilleke KS and Siriwardena AK: Systematic review of carbohydrate antigen (CA 19-9) as a biochemical marker in the diagnosis of pancreatic cancer. Eur J Surg Oncol 33: 266-270, 2007.

14. Ni XG, Bai XF, Mao YL, et al: The clinical value of serum CEA, CA19-9, and CA242 in the diagnosis and prognosis of pancreatic cancer. Eur J Surg Oncol 31: 164-169, 2005.

15. Yin BW and Lloyd KO: Molecular cloning of the CA125 ovarian cancer antigen: identification as a new mucin, MUC16. J Biol Chem 276: 27371-27375, 2001.

16. Streppel MM, Vincent A, Mukherjee R, et al: Mucin 16 (cancer antigen 125) expression in human tissues and cell lines and correlation with clinical outcome in adenocarcinomas of the pancreas, esophagus, stomach, and colon. Hum Pathol 43: 1755-1763, 2012.

17. Zhou G, Niu L, Chiu D, He L and Xu K: Changes in the expression of serum markers CA242, CA199, CA125, CEA, TNF-alpha and TSGF after cryosurgery in pancreatic cancer patients. Biotechnol Lett 34: 1235-1241, 2012. 